\title{
BMJ Open Associations between prescribed Chinese herbal medicine and risk of hepatocellular carcinoma in patients with chronic hepatitis B: a nationwide population-based cohort study
}

\author{
Tzung-Yi Tsai, ${ }^{1,2,3}$ Hanoch Livneh, ${ }^{4}$ Tsung-Hsing Hung, ${ }^{5,6}$ I -Hsin Lin, ${ }^{7}$ \\ Ming-Chi Lu, ${ }^{6,8}$ Chia-Chou Yeh ${ }^{7,9}$
}

To cite: Tsai T-Y, Livneh $\mathrm{H}$, Hung $\mathrm{T}-\mathrm{H}$, et al. Associations between prescribed Chinese herbal medicine and risk of hepatocellular carcinoma in patients with chronic hepatitis $B$ : a nationwide population-based cohort study. BMJ Open 2017;7: e014571. doi:10.1136/ bmjopen-2016-014571

- Prepublication history for this paper is available online. To view these files please visit the journal online (http://dx.doi.org/10.1136/ bmjopen-2016-014571).

Received 4 October 2016 Revised 7 December 2016 Accepted 21 December 2016

CrossMark

For numbered affiliations see end of article.

Correspondence to Professor Chia-Chou Yeh; yehcc0530@gmail.com

\section{ABSTRACT}

Objective: Patients with chronic hepatitis B (CHB) are reported to exhibit higher risk of subsequent hepatocellular carcinoma (HCC). However, it remains unclear if Chinese herbal medicine (CHM), an important category of traditional Chinese medicine (TCM), may lower HCC risk in this population. So this study aimed to investigate the effects of CHM on HCC risk among patients with $\mathrm{CHB}$.

Methods: This cohort study used the Taiwanese National Health Insurance Research Database to identify 21020 newly diagnosed patients with CHB from 1998 to 2007. Among them, 8640 received CHM products after CHB onset (CHM users), and the remaining 12380 patients were designated as a control group (non-CHM users). All enrolees were followed until the end of 2012 to measure the incidence rate and HR of HCC.

Results: During 15 years of follow-up, $371 \mathrm{CHM}$ users and 958 non-CHM users developed HCC, representing an incidence rate of $5.28 \%$ and $10.18 \%$ per 1000 person-years, respectively. CHM users had significantly lower HCC risk compared with non-CHM users (adjusted HR=0.63, 95\% $\mathrm{Cl} 0.56$ to 0.72 ). The predominant effect was observed in those receiving $\mathrm{CHM}$ products for more than 180 days (adjusted $\mathrm{HR}=0.52$ ). Some CHM products, such as Hedyotis diffusa, Scutellaria barbata, Rehmannia glutinosa, Isatis tinctoria, Yi Guan Jian, Xiao Chai Hu Tang, Wu Ling San and Gan Lu Yin, were significantly associated with lower risk of HCC.

Conclusions: The use of CHM was associated with a significantly reduced HCC risk in patients with $\mathrm{CHB}$, which supports the integration of TCM with CHM into clinical practice to influence a favourable prognosis.

\section{INTRODUCTION}

Hepatitis B virus (HBV) infection is a serious global public health concern. Chronic hepatitis B (CHB) infection can lead to acute and chronic hepatitis, liver cirrhosis, liver cancer

\section{Strengths and limitations of this study}

- The major strengths of this study included the application of retrospective cohort design as well as nationwide administrative database, which could decrease recall and selection bias and further provide stronger causal relationship than case-control or cross-sectional design.

- This is the first report to identify the therapeutic efficacy of Chinese herbal medicine on the subsequent risk of hepatocellular carcinoma among patients with chronic hepatitis $B$.

- Findings were beneficial for healthcare providers in guiding more effective treatment strategies to improve the clinical outcomes, and also a basis for further pharmacological investigations.

- Coding error of disease is a possibility while using the administrative database.

and liver failure. Approximately 2 billion people worldwide are reportedly infected with $\mathrm{HBV}$, and more than 350 million are chronic carriers. ${ }^{1}$ Vaccines against HBV have successfully reduced the incidence of HBV in younger generations. Nucleos(t)ide analogue therapy effectively suppresses HBV replication by inhibiting HBV polymerase. ${ }^{2}$ Treatment with nucleos(t)ide analogues has been reported to delay disease progression in patients with CHB. ${ }^{3} 4$ A meta-analysis reported that the use of nucleoside analogue therapy was associated with reduced risk of HCC in patients with CHB infection. ${ }^{5}$ However, more than 350 million patients are still infected with HBV worldwide. ${ }^{6}$ CHB infection causes hepatitis and also leads to hepatic decompensation, cirrhosis and hepatocellular carcinoma (HCC). ${ }^{6} 7$

The use of complementary and complementary medicine (CAM) is commonly practiced worldwide for preventive and 
therapeutic purposes. Traditional Chinese medicine (TCM) has been widely used in Asia for more than 2000 years. At present, TCM serves as an established segment of the public health system in China and Taiwan. In recent years, TCM has been gaining interest and acceptance as a form of alternative or complementary medicine in Western countries, particularly in the supportive and palliative care of patients with cancer. In 2007, $\$ 33.9$ million was spent by adults in the USA on CAM. ${ }^{8}$ An estimated 1.5 billion people now use Chinese herbal medicine (CHM), an important category of TCM, for the treatment of various diseases, including chronic HBV infection, worldwide. ${ }^{9}$ CHM is used as a treatment adjunct or alternative to anti-HBV drugs and accounts for $30 \%$ to $50 \%$ of the total medicine consumption for CHB treatment. ${ }^{10}$ It is also used by $19 \%$ of patients treated for liver cancer in Taiwan. ${ }^{11}$ Owing to its low cost and low toxicity, about $80 \%$ of patients with CHB in China and Taiwan have received CHM treatment. ${ }^{12}$

A number of clinical trials have been performed to assess the therapeutic efficacy and safety of CHM products. ${ }^{1} 1314$ On the other hand, a recent cohort study reported that adjunctive therapy with CHM may improve the survival of patients with liver cancer. ${ }^{15}$ Although nucleoside analogue therapy is associated with reduced risk of HCG in patients with $\mathrm{CHB}$ virus infection and experience fewer side effects, in many cases the drug resistance and viral variation limit their efficacy as therapeutic agents for CHB. ${ }^{1}$ Therefore, development of novel antiviral drugs and more effective therapies for the treatment of CHB are urgently needed. Some Chinese herbal medicines, including Chinese herbal formulas, single herbs and their active ingredients, are frequently reported to have antiviral effects in basic or clinical studies. ${ }^{1}{ }^{10} 16$ The effects of TCM on CHB, both alone and in combination with medicines of western origin, may indeed be effective therapeutic agents for the treatment of CHB. ${ }^{1}$ However, no long-term population-based nationwide study has investigated associations between the use of CHM products and the risk of HCG among HBV-infected patients. Therefore, the purpose of this retrospective, observational nationwide study was to test our hypothesis that the use of CHM reduces HCC risk in patients with CHB.

\section{METHODS}

\section{Data sources}

This retrospective cohort study collected claims data from the Longitudinal Health Insurance Database (LHID) of the Taiwan National Health Insurance Administration, whose information is made available to Taiwanese researchers. Taiwan launched the single-payer National Health Insurance (NHI) programme in 1995 to remove financial barriers to medical care for all legal residents. As of 2010, over 99\% of Taiwan's population was enrolled in this programme. ${ }^{17}$ The LHID is a subdata set of the NHI programme and is made up of 1000000 randomly sampled people who were alive in 2000. For the present study, all medical records of these individuals were collected from 1997 to 2012. The use of a multistage stratified systematic sampling method ensured that no statistically significant differences regarding sex or age existed between the one million insured individuals and the general population. ${ }^{17}$ This database contains all NHI enrolment files, claims data and the registry for prescription drugs, providing comprehensive utilisation information for the patients covered by the insurance programme. To date, the authors of more than 300 published papers have used this de-identified secondary database for their studies.

\section{Ethical considerations}

Since the LHID files contained only de-identified secondary data, the review board waived the requirement for obtaining informed consent from the patients.

\section{Study population}

Selection of study participants is shown in figure 1. All diagnoses in the insurance claims data were coded according to the International Classification of Disease, Ninth Revision, Clinical Modification (ICD-9-CM). Inclusion criteria for the study cohort were patients aged 20 years or older with newly diagnosed CHB (ICD-9-CM codes: 070.2, 070.3 or V02.61) and treated with nucleos(t)ide analogues, including lamiduvine, adefovir, telbivudine, entevacir or tenofovir between 1998 and 2007. The index date was defined as the day of CHB diagnosis. To reduce concerns of disease misclassification, only those patients with at least three outpatient visits with consistent diagnoses or those being admitted to a hospital with a primary diagnosis of $\mathrm{CHB}$ within the observational period $(n=22896)$ were selected. A total of 1171 patients with $\mathrm{CHB}$ were then excluded because they had a prior diagnosis of HCC (ICD-9-CM: 155.0 and 155.2) as indicated by linking the patients with $\mathrm{CHB}$ to the catastrophic illness registry. In Taiwan, insured residents with major diseases (eg, cancer, autoimmune diseases, chronic renal failure) can apply for a catastrophic illness certificate that grants exemption from copayment. Those with a follow-up period $<3$ months were also excluded $(n=705)$. Following these exclusions, the data from 21020 patients with CHB were retained for analysis.

In Taiwan, only certified practitioners of Chinese medicine are entitled to prescribe CHM products. As the existing rule proposes, ${ }^{18}$ the frequency of patients' visits for TCM was used to verify the CHM exposure of each study participant. Patients with CHB who received CHM treatment for more than 30 days due to a diagnosis of $\mathrm{CHB}$, within the follow-up period, were designated as CHM users, whereas those treated for 30 days or less, after the initial diagnosis of $\mathrm{CHB}$ were designated as non-CHM users. Follow-up person-years (PYs) of the non-CHM users were determined by calculating the 
Figure 1 Flow chart of selection and follow-up of study participants. $\mathrm{CHB}$, chronic hepatitis B; CHM, Chinese herbal medicine; HCC, hepatocellular carcinoma; NHI, National Health Insurance.
22,896 patients aged $20-80$ years with newly diagnosed $\mathrm{CHB}$ treated with nucleos(t)ide analogues between 1998 and 2007

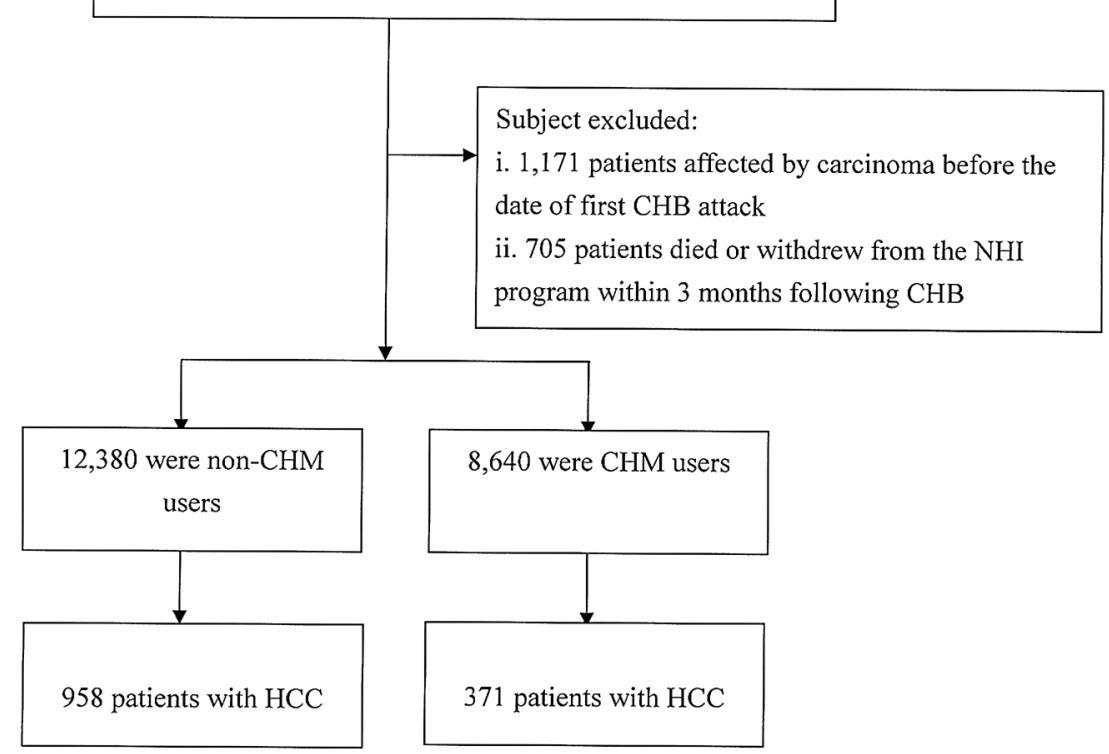

time interval from the index date to the earliest diagnosis of HCC, date of withdrawal from the insurance plan or study end-date of 31 December 2012, whichever of these dates came first. PYs of the CHM users were calculated from the initiation of receiving CHM products and were corrected by immortal time bias (selection bias), as previously described. ${ }^{19}$

\section{Demographic characteristics and comorbidities}

Demographic characteristics evaluated in this study included age, gender, income for estimating insurance payments and urbanisation level of participants' residential areas. Participants monthly incomes were stratified into three levels: $\leq$ New Taiwan Dollar (NTD) 17880 , NTD 17881 -NTD 43900, and ZNTD 43901. Urbanisation was divided into three levels by population: urban (levels 1-2), suburban (levels 3-4) and rural (levels 5-7) areas. Level 1 refers to the 'most urbanised' and level 7 refers to the 'least urbanised' communities, as described previously. ${ }^{20}$ Baseline comorbidities were evaluated by using the established Charlson-Deyo comorbidity index, ${ }^{21}$ which is based on individual medical records 1 year prior to initial cohort entry. In addition, medication usage was stratified into two groups according to whether the participants had received the nucleos $(\mathrm{t})$ ide analogues for more than 1 year after the index date.

\section{Statistical analysis}

Differences in demographic characteristics and comorbidities of CHM users versus non-CHM users were analysed using the $\chi^{2}$ test and Student's t-test. Cox proportional hazards regression analysis was applied to compute the HR with $95 \%$ CIs of HCC risk in association with CHM use. To test the robustness of the association between CHM use and HCC risk, CHM users were divided into two subgroups, one group that had received CHM products for 30-180 days and another group that had used CHM products for more than 180 days. We also used the Kaplan-Meier method to estimate cumulative risk of HCC between groups, and tested the difference with the log-rank test. Analyses stratified by age and gender were conducted using the Cox proportional hazards regression model to assess the relative risk of HCC between CHM users and non-CHM users. The proportional-hazards assumption was verified using plots of $\log (-\log$ (survival function)) versus $\log$ (time) and Schoenfeld residuals versus time. All statistical analyses were conducted using SAS V.9.3 (SAS Institute, Cary, North Carolina, USA), and $\mathrm{p}<0.05$ was established as statistical significance.

\section{RESULTS}

A total of 21020 patients with $\mathrm{CHB}$ were identified in the national insurance database. Of these, 8640 were CHM users and 12380 were non-CHM users. Table 1 shows the basic characteristics between groups. Compared to non-CHM users, CHM users were more likely to be women, younger, with lower monthly income and Charlson Comorbidity Index (CCI) scores and did not receive medications after the onset of $\mathrm{CHB}$ (all $\mathrm{p}<0.001)$.

A total of 1329 HCC events occurred among all study participants, including 371 non-CHM users and 958 CHM users, during follow-up of 70203.05 and 94122.45 PYs, respectively. Therefore, the incidence rate of HCC was lower in CHM users than in non-CHM users $(5.28$ vs 10.18 per $1000 \mathrm{PYs})$, with an adjusted HR of $0.63(95 \%$ CI 0.56 to 0.72 ; table 2). CHM use for more than 
Table 1 Demographic data and comorbidity comparison of the study participants

\begin{tabular}{|c|c|c|c|}
\hline Variables & $\begin{array}{l}\text { CHM } \\
\text { non-users } \\
\mathrm{N}=12380(\%)\end{array}$ & $\begin{array}{l}\text { CHM users } \\
\mathrm{N}=8640(\%)\end{array}$ & p Value \\
\hline Age, (years) & & & $<0.001$ \\
\hline$<=50$ & 7985 (64.5) & $6380(73.8)$ & \\
\hline$>50$ & 4395 (35.5) & 2260 (26.2) & \\
\hline Mean (SD, SD) & $45.69 \pm 14.09$ & $42.13 \pm 13.17$ & $<0.001$ \\
\hline Gender & & & $<0.001$ \\
\hline Female & 3741 (30.2) & 3966 (45.9) & \\
\hline Male & 8639 (69.8) & $4674(54.1)$ & \\
\hline Monthly income & & & 0.52 \\
\hline Low & 5860 (47.3) & 4090 (47.3) & \\
\hline Median & $5700(46.0)$ & 4011 (46.4) & \\
\hline High & $820(6.6)$ & $539(6.2)$ & \\
\hline Residential area & & & 0.23 \\
\hline Urban & 7084 (57.2) & $5011(58.0)$ & \\
\hline Suburban & 2085 (16.8) & 1478 (17.1) & \\
\hline Rural & 3211 (25.9) & 2151 (24.9) & \\
\hline Medication & & & $<0.001$ \\
\hline usage & & & \\
\hline Yes & $736(5.9)$ & $372(4.3)$ & \\
\hline No & $11644(94.1)$ & 8268 (95.7) & \\
\hline $\mathrm{CCl}$ & & & $<0.001$ \\
\hline Mean (SD) & $5.69 \pm 11.0$ & $4.79 \pm 9.1$ & \\
\hline
\end{tabular}

180 days was associated with a predominantly reduced risk of subsequent HCC by a magnitude of $52 \%$ (95\% CI 0.43 to 0.62 ). Figure 2 presents the Kaplan-Meier estimates of cumulative incidence across the three groups during the 15-year follow-up period, after adjusting for patients' age, sex, medication usage, CCI scores and urbanisation levels. The cumulative incidence of HCC for those receiving CHM treatment for more than 180 days was significantly lower than for those not receiving CHM (log-rank test, $\mathrm{p}<0.001)$.

Regarding gender-specific risk of HCC, both female and male CHM users showed significantly decreased risk of HCC, with an adjusted HR of 0.46 (95\% CI 0.38 to 0.61 ) and 0.70 (95\% CI 0.62 to 0.82 ), respectively (table 3 ). A significant interaction between age and sex was noted in association with CHM use. Therefore, a stratified analysis by age and sex was performed to determine the effect of CHM on HCC risk (table 3). Collectively, significant beneficial effects of CHM were observed in younger patients with $\mathrm{CHB}$, regardless of their sex. In men, decreases in adjusted HR were greater for CHM users aged $\leq 50$ years (adjusted HR $=0.69$, 95\% CI 0.56 to 0.85 ); in women, lower risk of HCC was observed in patients with $\mathrm{CHB}$ aged $\leq 50$ years (adjusted HR $=0.33,95 \%$ CI 0.21 to 0.55 ).

Table 4 lists the most commonly prescribed CHM products for treating CHB, including the HR of the 10 most commonly prescribed single-herb and multiherb products. Eight herbal products (Hedyotis diffusa, Scutellaria barbata, Rehmannia glutinosa, Isatis tinctoria, Yi Guan Jian, Xiao Chai Hu Tang, Wu Ling San, Gan Lu Yin) were associated with significantly lower risk of HCC.

\section{DISCUSSION}

According to a comprehensive literature review, this is the first population-based retrospective cohort study that provides solid evidence of the association between CHM use and HCC risk among patients with CHB treated with nucleos $(\mathrm{t})$ ide analogues. Results of the present study reveal that usage of prescribed CHM products by a large number of patients after CHB diagnosis is associated with significantly reduced HCC risk by $37 \%$.

Although medical guidelines do not currently recommend that complementary and/or alternative medicine be used in treating patients with CHB; CHM products have been commonly used worldwide for treating various illnesses. A previous study conducted in Taiwan reported that the overall prevalence of insurancecovered TCM use in outpatient services among patients with liver cancer was 21\%. ${ }^{11}$ Other studies have reported that $11 \%$ of women with early-stage breast cancer, ${ }^{22} 24 \%$ of patients with asthma, ${ }^{23}{ }^{24} 5 \%$ of patients receiving fracture treatment ${ }^{25}$ and $52.6 \%$ of patients with prostate cancer had used CHM to relieve symptoms or otherwise improve their quality of life. ${ }^{26}$ As with these diseases, the limitations of Western medicine in curing CHB appears to drive many patients to seek alternative treatments. We investigated the overall effects of prescribed CHM use on HCC risk in patients with $\mathrm{CHB}$ and explored the influence of specific Chinese herbal formulas on HCC risk. Our findings showed that nearly half $(41.1 \%)$ of the patients with CHB in Taiwan used CHM after CHB onset. However, before this study was conducted, little evidence existed to determine the efficacy of CHM in retarding CHB progression and HCC risk. Nucleos(t)ide analogue therapy effectively suppresses HBV replication

Table 2 Crude and adjusted HR of HCC for patients with $\mathrm{CHB}$ with and without $\mathrm{CHM}$ usage

\begin{tabular}{llllll}
\hline Patient group & Event & PYs & Incidence & Crude HR (95\% Cl) & Adjusted HR (95\% Cl) $^{*}$ \\
\hline CHM non-users & 958 & 94122.45 & 10.18 & 1 & 1 \\
CHM users & 371 & 70203.05 & 5.28 & $0.52(0.46$ to 0.58$)$ & $0.63(0.56$ to 0.72$)$ \\
CHM use within 30-180 days & 238 & 38879.55 & 6.12 & $0.59(0.52$ to 0.68$)$ & $0.73(0.63$ to 0.83$)$ \\
CHM use lasted for more than 180 days & 133 & 31323.50 & 4.25 & $0.43(0.36$ to 0.51$)$ & $0.52(0.43$ to 0.62$)$ \\
\hline
\end{tabular}

Incidence rate is per $1000 \mathrm{PYs}$.

*Model adjusted for age, gender, urbanisation level, monthly income, medication usage and $\mathrm{CCl}$ scores.

$\mathrm{CCl}$, Charlson Comorbidity Index; CHB, chronic hepatitis B; CHM, Chinese herbal medicine; HCC, hepatocellular carcinoma; PYs, person-years. 
Figure 2 Cumulative incidence of $\mathrm{HCC}$ for patients with $\mathrm{CHB}$ with and without $\mathrm{CHM}$ usage during the 15-year follow-up period (Log-rank test, $\mathrm{p}<0.001)$. CHB, chronic hepatitis $\mathrm{B}$; $\mathrm{CHM}$, Chinese herbal medicine; HCC, hepatocellular carcinoma; TCM, traditional Chinese medicine.

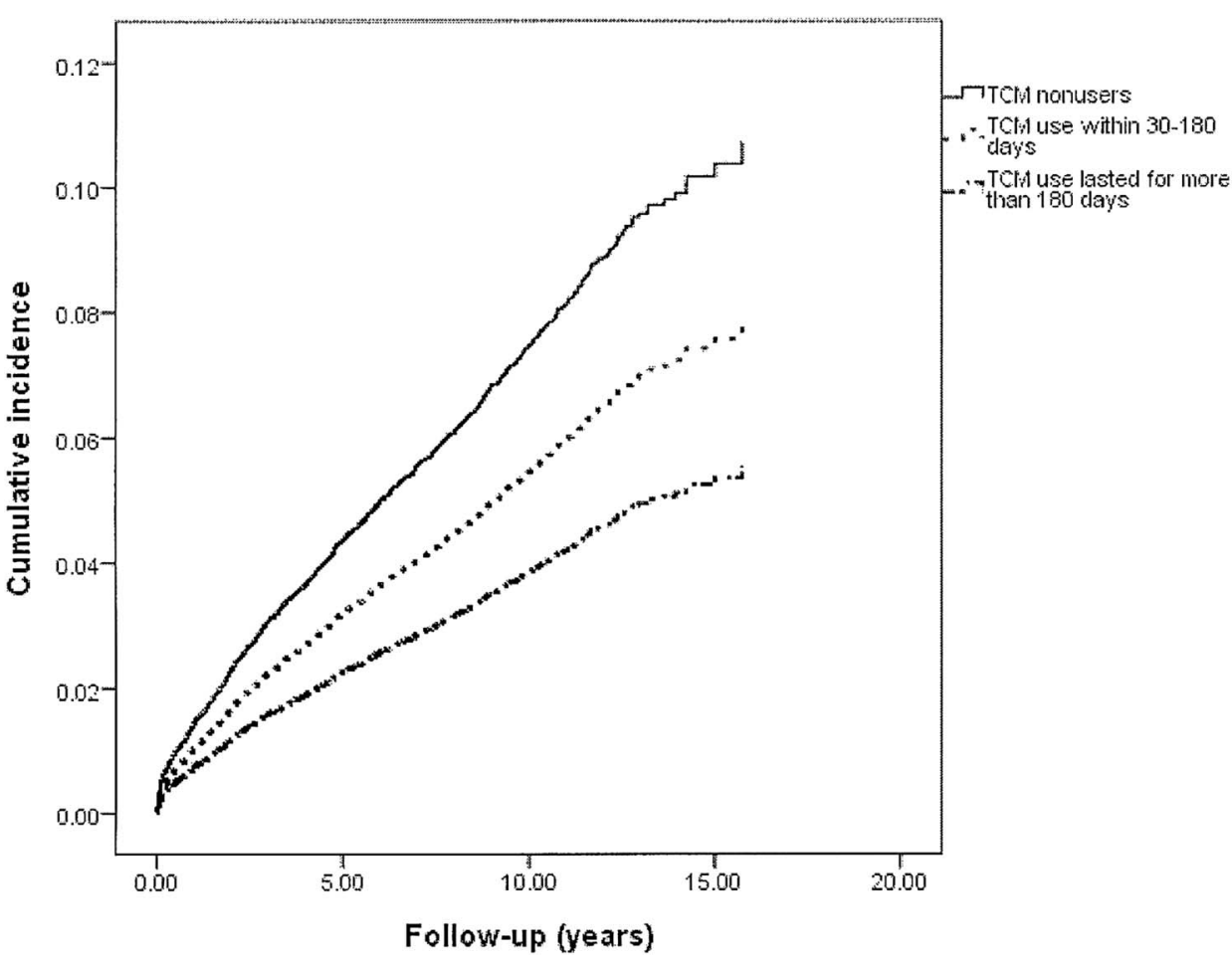

by inhibiting HBV polymerase. ${ }^{2}$ Treatment with nucleos (t)ide analogues has been reported to delay disease progression in patients with $\mathrm{CHB} .{ }^{27}$ In addition, nucleos $(\mathrm{t})$ ide analogue therapy has been reported to be associated with reduced risk of HCC development and recurrence. ${ }^{28} \mathrm{~A}$ meta-analysis reported that use of nucleos $(\mathrm{t})$ ide analogues was associated with reduced long-term risk of HCG in patients with $\mathrm{CHB} .^{5}$

In the present study, we found that the use of CHM was associated with reduced risk of HCC in patients with CHB treated with nucleos(t)ide analogues (adjusted $\mathrm{HR}=0.63$ ) compared to risk in control participants without CHM use. Results of the present study demonstrated further that the association between CHM use and subsequent risk of HCC among patients with $\mathrm{CHB}$ diminished with duration of use. The adjusted HR associated with CHM use were 0.73 for a treatment duration of 30-180 days, and 0.52 for treatment duration longer than 180 days. Several other studies have reported that CHM improved liver function and enhanced $\mathrm{HBeAg}$ and HBsAg seroconversion rates as well as HBV DNA clearance rates, ${ }^{1}$ which was associated with immune system response, ${ }^{29} 30$ liver fibrosis and cirrhosis, ${ }^{31} 32$ and HBV infection. ${ }^{1}$ Four $\mathrm{HBV}$ proteins, including the $\mathrm{X}$ protein $(\mathrm{HBx})$, have been implicated in the development of HCC. ${ }^{33-35}$ Other studies have also shown that TCM is likely to suppress HBx-mediated carcinogenesis and metastasis. $^{36} 37$

In the present study, the association between use of CHM and risk of HCC in patients with CHB diminished with age. The adjusted HRs associated with use of CHM were $0.46,0.33$ and 0.55 , for male patients with $\mathrm{CHB}$ of all ages, younger than 50 years and older than 50 years, respectively. This age-related interaction was likely due

\begin{tabular}{|c|c|c|c|c|c|c|c|c|}
\hline \multirow[b]{2}{*}{ Variables } & \multicolumn{3}{|c|}{ CHM non-users } & \multicolumn{3}{|c|}{ CHM users } & \multirow[b]{2}{*}{ Crude HR $(95 \% \mathrm{Cl})$} & \multirow[b]{2}{*}{ Adjusted HR (95\% Cl) } \\
\hline & Case & PYs & Incidence & Case & PYs & Incidence & & \\
\hline \multicolumn{9}{|l|}{ Female } \\
\hline$<=50$ & 56 & 16383.85 & 3.42 & 27 & 23273.49 & 1.16 & $0.34(0.22$ to 0.54$)$ & $0.33^{*}(0.21$ to 0.55$)$ \\
\hline$>50$ & 169 & 11316.90 & 14.93 & 69 & 8790.53 & 7.85 & $0.53(0.40$ to 0.69$)$ & $0.55^{*}(0.40$ to 0.73$)$ \\
\hline All & 225 & 27700.75 & 8.12 & 96 & 32064.02 & 2.99 & $0.37(0.29$ to 0.47$)$ & $0.46 \dagger(0.38$ to 0.61$)$ \\
\hline \multicolumn{9}{|l|}{ Male } \\
\hline$<=50$ & 312 & 47917.38 & 6.51 & 143 & 29922.58 & 4.78 & $0.73(0.60$ to 0.89$)$ & $0.69^{*}(0.56$ to 0.85$)$ \\
\hline$>50$ & 421 & 18504.33 & 22.75 & 132 & 8216.45 & 16.07 & $0.70(0.58$ to 0.84$)$ & $0.71^{*}(0.58$ to 0.86$)$ \\
\hline All & 733 & 66421.71 & 11.03 & 275 & 38139.03 & 7.21 & 0.65 (0.57 to 0.75$)$ & $0.70+(0.62$ to 0.82$)$ \\
\hline
\end{tabular}

Incidence rate is per 1000 PYs.

*Model adjusted for urbanisation level, monthly income, medication usage and $\mathrm{CCl}$ scores.

†Model adjusted for age, urbanisation level, monthly income, medication usage and $\mathrm{CCI}$ scores.

$\mathrm{CCI}$, Charlson Comorbidity Index; CHB, chronic hepatitis B; CHM, Chinese herbal medicine; PYs, person-years. 
Table 4 Risk of HCC in relation to the top 10 used single herb and multiherb products for patients with CHB

\begin{tabular}{|c|c|c|c|c|c|c|}
\hline Chinese herbal name & Scientific name & $\begin{array}{l}\text { Frequency of } \\
\text { prescriptions }\end{array}$ & $\begin{array}{l}\text { Average } \\
\text { duration (day) }\end{array}$ & $\begin{array}{l}\text { Daily } \\
\text { dose (g) }\end{array}$ & $\begin{array}{l}\text { Crude HR } \\
(95 \% \mathrm{Cl})\end{array}$ & $\begin{array}{l}\text { Adjusted HR* } \\
(95 \% \mathrm{Cl})\end{array}$ \\
\hline \multicolumn{7}{|l|}{ Single-herb products } \\
\hline Bai Hua She She Cao & Hedyotis diffusa & 466 & 7.9 & 16.4 & 0.37 (0.19 to 0.45$)$ & 0.38 (0.21 to 0.52$)$ \\
\hline Ban Zhi Lian & Scutellaria barbata & 596 & 6.9 & 8.1 & $0.30(0.24$ to 0.41$)$ & 0.29 (0.23 to 0.43$)$ \\
\hline Hai Piao Xiao & Endoconcha Sepiae & 388 & 11.1 & 13.3 & 1.01 (0.41 to 1.63$)$ & 1.04 (0.48 to 1.64$)$ \\
\hline Hu Zhang & Fallopia japonica & 452 & 8.9 & 6.5 & $0.58(0.24$ to 1.14$)$ & 0.57 (0.32 to 1.16$)$ \\
\hline Xiang Fu & Cyperus rotundus & 335 & 7.5 & 6.4 & 0.59 (0.20 to 1.38$)$ & $0.63(0.12$ to 1.39$)$ \\
\hline Yin Chen Hao. & Artemisia capillaris & 283 & 9.1 & 8.7 & 0.69 (0.36 to 1.10$)$ & 0.72 (0.37 to 1.12$)$ \\
\hline Shan Zha & Crataegi Fructus & 201 & 8.3 & 8.5 & 0.97 (0.31 to 1.89$)$ & 0.97 (0.36 to 1.93$)$ \\
\hline Sheng Di Huang & Rehmannia glutinosa & 374 & 11.5 & 5.6 & 0.27 (0.11 to 0.39$)$ & 0.35 (0.24 to 0.47$)$ \\
\hline Ban Lan Gen & Isatis tinctoria & 265 & 11.4 & 7.4 & $0.51(0.16$ to 0.75$)$ & $0.54(0.20$ to 0.81$)$ \\
\hline Tian Hua Fen & Trichosanthis kirilowii & 369 & 10.4 & 4.2 & 1.07 (0.66 to 1.63$)$ & 1.04 (0.58 to 1.54$)$ \\
\hline \multicolumn{7}{|l|}{ Multiherb products } \\
\hline Pin yin nomenclature & & & 8.7 & 6.5 & & \\
\hline Yi Guan Jian & & 293 & 6.9 & 2.1 & $0.43(0.26$ to 0.65$)$ & 0.65 (0.24 to 0.72$)$ \\
\hline Xiao Chai Hu Tang & & 695 & 8.5 & 7.3 & 0.44 (0.18 to 0.59$)$ & 0.53 (0.21 to 0.76$)$ \\
\hline Wu Ling San & & 233 & 9.1 & 5.8 & 0.75 (0.64 to 0.93$)$ & 0.82 (0.69 to 0.98$)$ \\
\hline Jia Wei Xiao Yao San & & 1317 & 7.1 & 7.6 & 0.82 (0.68 to 1.12$)$ & 0.94 (0.66 to 1.15$)$ \\
\hline Gan Lu Hsiao Tu Tan & & 541 & & 8.5 & 0.94 (0.75 to 1.28$)$ & 0.95 (0.79 to 1.27$)$ \\
\hline Gan Lu Yin & & 226 & 6.6 & 6.5 & 0.35 (0.19 to 0.49$)$ & 0.50 (0.24 to 0.76$)$ \\
\hline Chai Hu Ching Kan Tang & & 316 & 7.5 & 4.2 & $0.79(0.40$ to 1.20$)$ & 0.81 (0.46 to 1.21$)$ \\
\hline Chai Hu Shu Gan Tang & & 236 & 8.4 & 6.9 & 0.96 (0.74 to 1.16$)$ & 0.98 (0.76 to 1.18$)$ \\
\hline Yin chen Wu Lin San & & 1032 & 7.2 & 4.0 & $0.97(0.75$ to 1.11$)$ & 1.01 (0.74 to 1.19$)$ \\
\hline Long Dan Xie Gan Tang & & 241 & 6.3 & 5.2 & 0.76 (0.52 to 0.99$)$ & 0.83 (0.64 to 1.06$)$ \\
\hline
\end{tabular}


to an increased probability of HCC in patients with CHB of advanced age. According to a previous study of the natural history of patients with CHB in Taiwan, the risk of HCC in patients with CHB remains low before age 40 years, begins to rise during the 40 s and increases significantly after age 50 years. $^{7}$ A meta-analysis also reported that the risk of $\mathrm{HCC}$ in patients with $\mathrm{CHB}$ remains low before the age of 50 years and increases significantly after that time. ${ }^{5}$ The probability of HCC risk increasing with advanced age may reflect the risk reduction associated with CHM use due to differential use of TCM among different age groups. However, this speculation requires further empirical testing.

CHB has been treated for centuries with CHM. An estimated 1.5 billion people use CHM, an important category of TCM, for the treatment of various diseases, including chronic HBV infection worldwide. ${ }^{9}$ Owing to its low cost and low toxicity, about $80 \%$ of patients with CHB in China rely on this approach. ${ }^{12}$ A number of clinical trials have been performed to assess the therapeutic efficacy and safety of CHM products in CHB treatment. For example, CHM combined with interferon or lamivudine significantly enhanced the antiviral activities of these agents and, accordingly, CHM was proven to have a beneficial impact on improving liver function. ${ }^{10}{ }^{12}$ At the same time, several studies have demonstrated that CHM use is beneficial in alleviating clinical symptoms, improving quality of life and immune function, preventing recurrence and metastasis, delaying tumour progression and prolonging survival of patients with hepatocarcinoma. ${ }^{38}$ Studies in China have also reported that CHM may have beneficial therapeutic effects on CHB. ${ }^{39-41}$

The present study has listed the most commonly used single-herb and multiherb products used in treating CHB in our large cohort (table 4). The prescription process for CHM differs from that of biomedical prescriptions in that there is no defined 1:1 relationship between the prescription and the disorder type, and the treatment of CHB may vary according to the symptoms and signs displayed by the patient. Although some of the herbs listed, such as Hedyotis diffusa, ${ }^{42} 43$ Scutellaria barbata, ${ }^{44}{ }^{45}$ Rehmannia glutinosa,${ }^{46}$ Isatis tinctoria, ${ }^{46} 47$ and Xiao Chai Hu Tang, ${ }^{4-50}$ have been investigated in the context of CHB or HCC, while other herbs such as Yi Guan Jian, Wu Ling San, and Gan Lu Yin have not been carried out. Thus, the products listed in table 4 may still be investigated in future basic and clinical research.

Findings of the present study have important clinical and research implications regarding the use of CHM in treating CHB. However, several limitations should be noted when interpreting the results of this study. First, we identified patients with CHB and HCC using ICD-9-CM codes rather than laboratory data, and misclassification is possible. To minimise this error, we selected participants with CHB only after they were recorded as having at least three outpatient visits and reporting consistent diagnoses or at least one inpatient admission. It should also be noted that the NHI of Taiwan randomly samples claims from hospitals, interviews patients and reviews medical charts to verify the accuracy of medical records. Second, information on drinking alcohol, social network relationships, coping strategies and resources, religious beliefs and educational level or the genotypes of HBV were unavailable from the claims data. Drinking alcohol is an important risk factor associated with HCC development in patients with CHB. The failure to adjust for the confounding effect of drinking alcohol could possibly lead to biased estimates of HCC risk in our sample. However, the sexstratified analysis supports an appreciably decreased risk of HCC for patients with CHB receiving CHM regardless of gender, while the alcohol drinking rates for women and men in Taiwan are $0.4 \%$ and $25 \%$, respectively. ${ }^{51}$ This observation suggests that the confounding effect of drinking alcohol was unlikely to compromise the results of this study. Third, we were unable to contact the enrolled patients directly regarding their use of CHM products due to the anonymity of identification numbers in the database. However, nearly $95 \%$ of the dose frequencies in Chinese herbs are typically used for only 1 week in clinical practice, so those who continued to receive the same prescription for a longer period of time were therefore likely to have used the prescribed medication. ${ }^{52}$ Additionally, prescriptions for medications issued before 1996 were not reflected in data analysis in the present study. This omission could possibly result in underestimating the cumulative frequencies and may have weakened the effect of the specified CHM products. Fourth, though the multivariate analysis applied in this study did consider the impact of nucleos $(t)$ ide analogue, to test the robustness of the present findings, a sensitivity analysis limited to those patients with CHB with CHM usage but without receiving any nucleos $(\mathrm{t})$ ide analogue was done and revealed that the protective benefits of CHM were still in effect (adjusted HR $=0.65 ; 95 \% \mathrm{CI}=0.55$ to 0.71 ). These limitations notwithstanding, the strengths of this study must also be acknowledged, including the immediate availability of data, the comprehensiveness of the database and the statistical power derived from the large-sized sample. In addition, this retrospective 15-year cohort study allowed us to examine in detail the associations between CHM usage and HCC risk, and the corresponding findings may serve as a reference for future studies.

In conclusion, the results of this 15-year follow-up cohort study suggest that the integration of CHM during treatment of CHB is associated with a $37 \%$ lower risk of developing HCC compared to risk among non-CHM users. Results of this study may serve as a reference for healthcare providers to help establish more effective therapeutic interventions to improve the prognosis of patients with CHB. 
Author affiliations

${ }^{1}$ Department of Medical Research, Dalin Tzuchi Hospital, The Buddhist Tzuchi Medical Foundation, Chiayi, Taiwan

2Department of Environmental and Occupational Health, College of Medicine, National Cheng Kung University, Tainan, Taiwan

${ }^{3}$ Department of Nursing, Tzu Chi College of Technology, Hualien, Taiwan

${ }^{4}$ Rehabilitation Counseling Program, Portland State University, Portland, Oregon, USA

${ }^{5}$ Division of Gastroenterology, Dalin Tzu Chi Hospital, Buddhist Tzu Chi

Foundation, Chiayi, Taiwan

${ }^{6}$ School of Medicine, Hualien, Taiwan

${ }^{7}$ School of Post-Baccalaureate Chinese Medicine, Tzu Chi University, Hualien, Taiwan

${ }^{8}$ Division of Allergy, Immunology and Rheumatology, Dalin Tzuchi Hospital,

The Buddhist Tzuchi Medical Foundation, Chiayi, Taiwan

${ }^{9}$ Department of Chinese Medicine, Dalin Tzuchi Hospital, The Buddhist Tzuchi Medical Foundation, Chiayi, Taiwan

Acknowledgements This study was supported by Buddhist Dalin Tzuchi Hospital (grant number DTCRD103(2)-E-05). Additionally, this study is based in part on data from the National Health Insurance Research Database provided by the Bureau of National Health Insurance, Department of Health and managed by the National Health Research Institutes. The interpretation and conclusions contained herein do not represent those of the Taiwan Bureau of National Health Insurance, Department of Health or the National Health Research Institutes.

Contributors TYT and THH contributed equally in this work. TYT and CCY were involved in study concept and design; acquisition of data; statistical analysis and interpretation of data; drafting of the manuscript. $\mathrm{HL}$ and THH: study concept and design and interpretation of data. IHL and MCL administrative, technical and material support. All authors approved the final version of the manuscript.

Funding The researcher received no specific grant form any funding agency in the public, commercial or not-for-profit sectors.

Competing interests None declared.

Ethics approval This study was approved by the Research Ethics Committee of Dalin Tzuchi Hospital No. B10004021-1.

Provenance and peer review Not commissioned; externally peer reviewed.

Data sharing statement No additional data are available.

Open Access This is an Open Access article distributed in accordance with the Creative Commons Attribution Non Commercial (CC BY-NC 4.0) license, which permits others to distribute, remix, adapt, build upon this work noncommercially, and license their derivative works on different terms, provided the original work is properly cited and the use is non-commercial. See: http:// creativecommons.org/licenses/by-nc/4.0/

\section{REFERENCES}

1. Qi FH, Wang ZX, Cai PP, et al. Traditional Chinese medicine and related active compounds: a review of their role on hepatitis $B$ virus infection. Drug Discov Ther 2013;7:212-24.

2. Yuen MF, Lai CL. Treatment of chronic hepatitis B: evolution over two decades. J Gastroenterol Hepatol 2011;26(Suppl 1):138-43.

3. Liaw YF, Sung JJ, Chow WC, et al. Lamivudine for patients with chronic hepatitis B and advanced liver disease. N Engl J Med 2004;351:1521-31.

4. Chang TT, Liaw YF, Wu SS, et al. Long-term entecavir therapy results in the reversal of fibrosis/cirrhosis and continued histological improvement in patients with chronic hepatitis B. Hepatology 2010;52:886-93.

5. Liu S, Zhang H, Gu C, et al. Associations between hepatitis B virus mutations and the risk of hepatocellular carcinoma: a meta-analysis. J Natl Cancer Inst 2009;101:1066-82.

6. Liaw YF, Chu CM. Hepatitis B virus infection. Lancet 2009;373:582-92

7. Chen CJ, Yang HI. Natural history of chronic hepatitis B REVEALed J Gastroenterol Hepatol 2011;26:628-38.

8. Nahin RL, Barnes PM, Stussman BJ, et al. Costs of complementary and alternative medicine (CAM) and frequency of visits to CAM practitioners: United States, 2007. Natl Health Stat Report 2009;30:1-14.

9. Dobos GJ, Tan L, Cohen $\mathrm{MH}$, et al. Are national quality standards for traditional Chinese herbal medicine sufficient? Current governmental regulations for traditional Chinese herbal medicine in certain Western countries and China as the Eastern origin country. Complement Ther Med 2005;13:183-90.

10. McCulloch M, Broffman M, Gao J, et al. Chinese herbal medicine and interferon in the treatment of chronic hepatitis $\mathrm{B}$ : a meta-analysis of randomized, controlled trials. Am J Public Health 2002;92: 1619-28.

11. Liao $\mathrm{YH}$, Lin CC, Li TC, et al. Utilization pattern of traditional Chinese medicine for liver cancer patients in Taiwan. BMC Complement Altern Med 2012;12:146.

12. Zhang L, Wang G, Hou W, et al. Contemporary clinical research of traditional Chinese medicines for chronic hepatitis B in China: an analytical review. Hepatology 2010;51:690-8.

13. Qu J, Yu Z, Li Q, et al. Blocking and reversing hepatic fibrosis in patients with chronic hepatitis $B$ treated by traditional Chinese medicine (tablets of biejia ruangan or RGT): study protocol for a randomized controlled trial. Trials 2014;15:438.

14. Shen WS, Yang HZ, Hong Q, et al. Two-year observation of the clinical efficacy in treating chronic hepatitis B Patients with Ganxian recipe and lamivudine. Chin J Integr Med 2005;11:5-10.

15. Liao $\mathrm{YH}$, Lin CC, Lai HC, et al. Adjunctive traditional Chinese medicine therapy improves survival of liver cancer patients. Liver Int 2015;35:2595-602.

16. Cui X, Wang $\mathrm{Y}$, Kokudo $\mathrm{N}$, et al. Traditional Chinese medicine and related active compounds against hepatitis $\mathrm{B}$ virus infection. Biosci Trends 2010;4:39-47.

17. National Health Insurance Research Database, Taiwan. http://nhird. nhri.org.tw/date_cohort.html (accessed May 2016).

18. Tsai TY, Li CY, Livneh $\mathrm{H}$, et al. Decreased risk of stroke in patients receiving traditional Chinese medicine for vertigo: a population-based cohort study. J Ethnopharmacol 2016;184:138-43.

19. Shariff SZ, Cuerden MS, Jain AK, et al. The secret of immortal time bias in epidemiologic studies. J Am Soc Nephrol 2008;19:841-3.

20. Liu CY, Hung YT, Chuang Y, et al. Incorporating development stratification of Taiwan townships into sampling design of large scale health interview survey. J Health Manag 2006;4:1-22.

21. Deyo RA, Cherkin DC, Ciol MA. Adapting a clinical comorbidity index for use with ICD-9-CM administrative databases. J Clin Epidemiol 1992;45:613-19.

22. Burstein $\mathrm{HJ}$, Gelber S, Guadagnoli E, et al. Use of alternative medicine by women with early-stage breast cancer. $N$ Engl J Med 1999;340:1733-9

23. Hung YC, Hung IL, Sun MF, et al. Integrated traditional Chinese medicine for childhood asthma in Taiwan: a nationwide cohort study. BMC Complement Altern Med 2014;14:389.

24. Blanc PD, Trupin L, Earnest G, et al. Alternative therapies among adults with a reported diagnosis of asthma or rhinosinusitis: data from a population-based survey. Chest 2001;120:1461-7.

25. Liao $\mathrm{HH}$, Yeh CC, Lin CC, et al. Prescription patterns of Chinese herbal products for patients with fractures in Taiwan: a nationwide population-based study. J Ethnopharmacol 2015;173:11-19.

26. Lin YH, Chen KK, Chiu JH. Use of Chinese medicine among prostate cancer patients in Taiwan: a retrospective longitudinal cohort study. Int J Urol 2011;18:383-6.

27. Liaw YF. Impact of hepatitis B therapy on the long-term outcome of liver disease. Liver Int 2011;31(Suppl 1):117-21.

28. Wu CY, Lin JT, Ho HJ, et al. Association of nucleos(t)ide analogue therapy with reduced risk of hepatocellular carcinoma in patients with chronic hepatitis B: a nationwide cohort study. Gastroenterology 2014;147:143-51.

29. Wu L, Li H, Zheng SZ, et al. Da-Huang-Fu-Zi-Tang attenuates liver injury in rats with severe acute pancreatitis. J Ethnopharmacol 2013;150:960-6.

30. Meng MB, Wen QL, Cui YL, et al. Meta-analysis: traditional Chinese medicine for improving immune response in patients with unresectable hepatocellular carcinoma after transcatheter arteria chemoembolization. Explore (NY) 2011;7:37-43.

31. Chen QL, Lu YY, Peng JH, et al. Dynamical regulation analysis identifies molecular mechanisms of Fuzheng-Huayu formula against hepatitis B-caused liver cirrhosis. Evid Based Complement Alternat Med 2015;2015:238495.

32. Teng L, Zhang J, Dai M, et al. Correlation between traditional Chinese medicine symptom patterns and serum concentration of zinc, iron, copper and magnesium in patients with hepatitis $B$ and associated liver cirrhosis. J Tradit Chin Med 2015;35:546-50.

33. Cromlish JA. Hepatitis B virus-induced hepatocellular carcinoma: possible roles for HBx. Trends Microbiol 1996;4:270-4. 
34. Kim CM, Koike K, Saito I, et al. HBx gene of hepatitis B virus induces liver cancer in transgenic mice. Nature 1991;351:317-20.

35. Su Q, Schröder $\mathrm{CH}$, Hofmann WJ, et al. Expression of hepatitis $\mathrm{B}$ virus $\mathrm{X}$ protein in $\mathrm{HBV}$-infected human livers and hepatocellular carcinomas. Hepatology 1998;27:1109-20.

36. Lin HJ, Kao ST, Siao YM, et al. The Chinese medicine sini-san inhibits $\mathrm{HBx}$-induced migration and invasiveness of human hepatocellular carcinoma cells. BMC Complement Altern 2015;15:348.

37. Su YC, Lin IH, Siao YM, et al. Modulation of the tumor metastatic microenvironment and multiple signal pathways by prunella vulgaris in human hepatocellular carcinoma. Am J Chin 2016;44:835-49.

38. Hu B, Wang SS, Du Q. Traditional Chinese medicine for prevention and treatment of hepatocarcinoma: from bench to bedside. World $J$ Hepatol 2015;7:1209-32.

39. Yu JH, Shi JP, Wu J, et al. [Efficacy and safety of lamivudine plus adefovir combination therapy and entecavir monotherapy for chronic hepatitis B patients]. Zhonghua Gan Zang Bing Za Zhi 2011;19:88-92.

40. Chen $\mathrm{T}$, Chen WH. [Advances of combined treatment for chronic hepatitis B with lamivudine]. Zhong Xi Yi Jie He Xue Bao 2003;1:146-50.

41. Feng $\mathrm{H}$, Zhang $\mathrm{YH}$. Effect of Chinese medicine therapy for strengthening-pi and nourishing-shen in preventing lamivudine induced YMDD mutation and its immunologic mechanism. Chin J Integr Med 2010;16:19-22.

42. Lee HZ, Bau DT, Kuo CL, et al. Clarification of the phenotypic characteristics and anti-tumor activity of hedyotis diffusa. Am J Chin Med 2011;39:201-13.

43. Chen XZ, Cao ZY, Chen TS, et al. Water extract of hedyotis diffusa willd suppresses proliferation of human HepG2 cells and potentiates the anticancer efficacy of low-dose 5 -fluorouracil by inhibiting the CDK2-E2F1 pathway. Oncol Rep 2012;28:742-8.
44. Chan JY, Tang PM, Hon PM, et al. Pheophorbide a, a major antitumor component purified from scutellaria barbata, induces apoptosis in human hepatocellular carcinoma cells. Planta Med 2006;72:28-33.

45. Juan M, Minhu C, Yun D, et al. Enhancing the efficacy of photodynamic therapy by a Chinese herbal medicine for hepatocellular carcinoma. Cancer Biol Ther 2006;5: 1117-19.

46. Chao JC, Chiang SW, Wang CC, et al. Hot water-extracted Lycium barbarum and rehmannia glutinosa inhibit proliferation and induce apoptosis of hepatocellular carcinoma cells. World J Gastroenterol 2006;12:4478-84.

47. Du H, Zhang S, Song M, et al. Assessment of a flavonepolysaccharide based prescription for treating duck virus hepatitis. PLOS ONE 2016;11:e0146046.

48. Oka H, Yamamoto $\mathrm{S}$, Kuroki T, et al. Prospective study of chemoprevention of hepatocellular carcinoma with Sho-saiko-to (TJ-9). Cancer 1995;76:743-9.

49. Lee JK, Kim JH, Shin HK. Therapeutic effects of the oriental herbal medicine sho-saiko-to on liver cirrhosis and carcinoma. Hepatol Res 2011:41:825-37.

50. Rino Y, Yukawa N, Yamamoto N. Does herbal medicine reduce the risk of hepatocellular carcinoma? World J Gastroenterol 2015;21:10598-603.

51. Hu JH, Chen MY, Yeh CT, et al. Sexual dimorphic metabolic alterations in hepatitis $\mathrm{C}$ virus-infected patients: a community-based study in a hepatitis $B /$ hepatitis $C$ virus hyperendemic area. Medicine (Baltimore) 2016;95:e3546.

52. Lai MN, Wang SM, Chen PC, et al. Population-based case-control study of Chinese herbal products containing aristolochic acid and urinary tract cancer risk. J Natl Cancer Inst 2010;102:179-86. 


\section{Correction}

Tsai T, Livneh H, Hung T, et al. Associations between prescribed Chinese herbal medicine and risk of hepatocellular carcinoma in patients with chronic hepatitis $\mathrm{B}$ : a nationwide population-based cohort study. BMJ Open 2017;7:e014571. doi: 10.1136/ bmjopen-2016-014571

Affiliation 6 Is incomplete. It should read "School of Medicine, Tzu Chi University, 701 Jhongyang Road Section 3, Hualien 97004, Taiwan”.

Open Access This is an Open Access article distributed in accordance with the Creative Commons Attribution Non Commercial (CC BY-NC 4.0) license, which permits others to distribute, remix, adapt, build upon this work noncommercially, and license their derivative works on different terms, provided the original work is properly cited and the use is non-commercial. See: http://creativecommons.org/licenses/by-nc/4.0/

BMJ Open 2017;7:e014571corr1. doi:10.1136/bmjopen-2016-014571corr1

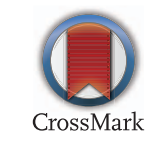

